\title{
Anharmonic vibrational properties in periodic systems: energy, electron-phonon coupling, and stress
}

\author{
Bartomeu Monserrat, ${ }^{1, *}$ N. D. Drummond, ${ }^{2}$ and R. J. Needs ${ }^{1}$ \\ ${ }^{1}$ TCM Group, Cavendish Laboratory, University of Cambridge, J. J. Thomson Avenue, Cambridge CB3 OHE, United Kingdom \\ ${ }^{2}$ Department of Physics, Lancaster University, Lancaster LAI 4YB, United Kingdom
}

(Received 1 March 2013; published 8 April 2013)

\begin{abstract}
A unified approach is used to study vibrational properties of periodic systems with first-principles methods and including anharmonic effects. Our approach provides a theoretical basis for the determination of phonondependent quantities at finite temperatures. The low-energy portion of the Born-Oppenheimer energy surface is mapped and used to calculate the total vibrational energy including anharmonic effects, electron-phonon coupling, and the vibrational contribution to the stress tensor. We report results for the temperature dependence of the electronic band gap and the linear coefficient of thermal expansion of diamond, lithium hydride, and lithium deuteride.
\end{abstract}

DOI: 10.1103/PhysRevB.87.144302

PACS number(s): 63.20.Ry, 63.20.kd, 65.40.De, 65.60.+a

\section{INTRODUCTION}

The atomic vibrations that are always present in condensed matter lead to important corrections to the static-lattice model of crystalline solids. The most common approach for evaluating such corrections is to include vibrational effects within the harmonic phonon approximation, ${ }^{1-3}$ which works well in many circumstances. However, anharmonic effects can be important in, for example, systems incorporating light atoms which have large vibrational amplitudes, systems with weak bonding such as hydrogen bonding, and systems at high temperatures. When the vibrations are present, their effect on the electrons can also become important. Electron-phonon interactions are central, for example, to the description of superconductivity.

Renormalization of the phonon degrees of freedom is an important idea underlying most treatments of phononphonon and electron-phonon interactions. For example, (i) the phonon frequencies as a function of temperature may be used to investigate the stability of crystals with unstable harmonic phonons, (ii) the change in phonon frequencies due to electron-phonon interactions may be used in the study of the temperature dependence of electronic band gaps, and (iii) the phonon frequencies at different volumes may be used within the quasiharmonic approximation to study thermal expansion.

The self-consistent phonon method was devised by Born and Hooton, ${ }^{4-7}$ and extended by Choquard ${ }^{8}$ and Werthamer. ${ }^{9}$ In the self-consistent phonon method, the phonon frequencies are renormalized by considering the atomic vibrations over a region about equilibrium which can include anharmonicity. For example, it is useful in systems with a harmonic instability, such as solid helium which forms under pressure, where including anharmonic terms leads to the correct physical picture in which the body-centered-cubic structure is stabilized. A historical overview of these developments is given by Klein and Horton. ${ }^{10}$

More recently, these ideas have been used in first-principles density functional theory (DFT) electronic structure calculations and have shown the occurrence of dynamically unstable phases at the harmonic level that are stabilized at finite temperature by means of anharmonicity. ${ }^{11}$ Souvatzis and co-workers ${ }^{12}$ and Hellman and co-workers ${ }^{13}$ have calculated renormalized phonons at finite temperature for systems with harmonic phonon instabilities. Antolin and co-workers ${ }^{14}$ presented a simpler and computationally less demanding method for solving this problem, using a harmonic fit to large-amplitude phonon displacements.

Thermal expansion arises from anharmonicity and is usually addressed within the quasiharmonic approximation, ${ }^{15}$ in which the phonon frequencies are taken to depend on the volume. The minimization of the free energy with respect to volume at a given temperature leads to the equilibrium volume at that temperature. This method has been very successful, but for systems with several lattice parameters it is computationally expensive to relax the structures at each volume.

Lattice vibrations also play a central role in electron-phonon interactions. The study of electron-phonon interactions within many-body perturbation theory is well established. ${ }^{16}$ The effects of electron-phonon interactions on the temperature dependence of band gaps are substantial, and they have been described within the harmonic approximation. ${ }^{17-19}$ Recent theoretical advances have enabled such calculations to be performed from first principles. ${ }^{20}$

In this paper we describe a unified approach for studying the effects of anharmonicity at zero and finite temperatures on quantities including phase stability, the total energy, electronphonon coupling leading to band gap renormalization, and thermal expansion.

The starting point of our method involves performing first-principles calculations of the low-energy portion of the Born-Oppenheimer $(\mathrm{BO})^{21}$ energy surface defined by the harmonic phonon coordinates, and exploring it out to large atomic displacements where the harmonic approximation is no longer accurate. This information is used to calculate anharmonic phonon free energies and the associated vibrational excited states using the vibrational self-consistent-field (VSCF) equations. ${ }^{22}$ We then use a perturbation theory constructed on the VSCF equations ${ }^{23}$ to obtain a second-order correction to the total vibrational free energy. This method has been used before in studying molecules ${ }^{22,24}$ and extended model systems, ${ }^{25}$ but, as far as we are aware, this is the first firstprinciples application to three-dimensional periodic solids. 
We have used the anharmonic wave functions to calculate quantum-mechanical expectation values of phonon-dependent quantities at zero and finite temperatures. For example, renormalization of the electronic eigenvalues due to their interaction with the phonons leads to the temperature dependence of the electronic band structure. Similarly, the vibrational stress tensor can be calculated and used to directly study thermal expansion.

The main computational cost in our approach is that of performing the electronic structure calculations to explore the BO energy surface. While performing these calculations, data for all quantities of interest can be accumulated, and the further analysis of particular quantities can be done with little additional computational cost.

We report results for the anharmonic energy, the temperature dependence of the thermal band gap, and the linear coefficient of thermal expansion of diamond, lithium hydride $\left(\mathrm{H}^{7} \mathrm{Li}\right)$, and lithium deuteride $\left(\mathrm{D}^{7} \mathrm{Li}\right)$. Our results are in good agreement with experiment and other theoretical approaches. As far as we are aware, no experimental or theoretical results for the temperature dependence of the band gap of lithium hydride have been published previously.

The remainder of this paper is arranged as follows. In Sec. II we present the theoretical framework used to study anharmonicity and in Sec. III we extend the formalism to the evaluation of general phonon expectation values. In Sec. IV we describe our computational approach. Our results are presented in Sec. V and we draw our conclusions in Sec. VI. All equations are given in Hartree atomic units, in which the Dirac constant, the electronic charge and mass, and $4 \pi$ times the permittivity of free space are unity $\left(\hbar=|e|=m_{\mathrm{e}}=4 \pi \epsilon_{0}=1\right)$.

\section{ANHARMONIC TOTAL ENERGY}

\section{A. BO approximation}

Within the BO approximation ${ }^{21}$ the electronic and vibrational degrees of freedom are treated separately. The atomic positions parametrize a family of electronic Hamiltonians $\hat{H}_{\mathrm{el}}(\mathbf{R})$ labeled by the collective nuclear position variable $\mathbf{R}$. Each of these Hamiltonians leads to the electronic energy $E_{\mathrm{el}}$ and wave function $|\Psi\rangle$ according to $\hat{H}_{\mathrm{el}}(\mathbf{R})|\Psi(\mathbf{R})\rangle=$ $E_{\mathrm{el}}(\mathbf{R})|\Psi(\mathbf{R})\rangle$. In the following we consider only the electronic ground state.

The nuclear or vibrational Hamiltonian $\hat{H}_{\text {vib }}$ is

$$
\hat{H}_{\mathrm{vib}}=\sum_{\mathbf{R}_{p}, \alpha}-\frac{1}{2 m_{\alpha}} \nabla_{p \alpha}^{2}+E_{\mathrm{el}}(\mathbf{R})
$$

where the $\mathbf{R}_{p}$ are the position vectors of the unit cells that make up the periodic supercell, $\alpha$ labels the different atoms within a unit cell, and $m_{\alpha}$ is the mass of atom $\alpha$. In this context, the electronic eigenvalue as a function of atomic position $E_{\mathrm{el}}(\mathbf{R})$ is called the BO surface.

\section{B. Harmonic approximation}

Within the harmonic approximation $(\mathrm{HA})^{1-3}$ the BO energy surface is approximated as a quadratic function of the atomic displacement coordinates $\mathbf{u}_{p \alpha}=\mathbf{r}_{p \alpha}-\mathbf{r}_{p \alpha}^{0}$,

$$
\begin{aligned}
\hat{H}_{\mathrm{vib}}= & \sum_{\mathbf{R}_{p}, \alpha}-\frac{1}{2 m_{\alpha}} \nabla_{p \alpha}^{2}+E_{\mathrm{el}}\left(\mathbf{R}^{0}\right) \\
& +\frac{1}{2} \sum_{\substack{\mathbf{R}_{p}, \alpha, i \\
\mathbf{R}_{p^{\prime}}, \alpha^{\prime}, j}} \frac{\partial^{2} E_{\mathrm{el}}\left(\mathbf{R}^{0}\right)}{\partial u_{p \alpha ; i} \partial u_{p^{\prime} \alpha^{\prime} ; j}} u_{p \alpha ; i} u_{p^{\prime} \alpha^{\prime} ; j},
\end{aligned}
$$

where $\mathbf{r}_{p \alpha}$ are the atomic positions, $\mathbf{r}_{p \alpha}^{0}$ the equilibrium atomic positions, and Latin indices $i$ and $j$ are used to label Cartesian directions. The HA often works very well because the nuclei are heavy and therefore only explore the neighborhood of their equilibrium positions. In the following we drop the constant energy term $E_{\mathrm{el}}\left(\mathbf{R}^{0}\right)$.

The matrix of force constants is defined as $D_{i \alpha ; j \alpha^{\prime}}\left(\mathbf{R}_{p}, \mathbf{R}_{p}^{\prime}\right)=\partial^{2} E_{\mathrm{el}}\left(\mathbf{R}^{0}\right) / \partial u_{p \alpha ; i} \partial u_{p^{\prime} \alpha^{\prime} ; j}, \quad$ and the dynamical matrix at the reciprocal space point $\mathbf{k}$ is

$$
D_{i \alpha ; j \alpha^{\prime}}(\mathbf{k})=\frac{1}{N_{p} \sqrt{m_{\alpha} m_{\alpha^{\prime}}}} \sum_{\mathbf{R}_{p}, \mathbf{R}_{p^{\prime}}} D_{i \alpha ; j \alpha^{\prime}}\left(\mathbf{R}_{p}, \mathbf{R}_{p}^{\prime}\right) e^{i \mathbf{k} \cdot\left(\mathbf{R}_{p}-\mathbf{R}_{p}^{\prime}\right)},
$$

where $N_{p}$ is the number of unit cells within the periodic supercell.

The vibrational Hamiltonian of Eq. (2) can be rewritten in terms of normal or phonon coordinates $q_{n \mathbf{k}}$, which are related to displacement coordinates by

$$
\begin{gathered}
u_{p \alpha ; i}=\frac{1}{\sqrt{N_{p} m_{\alpha}}} \sum_{n, \mathbf{k}} q_{n \mathbf{k}} e^{i \mathbf{k} \cdot \mathbf{R}_{p}} w_{\mathbf{k} n ; i \alpha}, \\
q_{n \mathbf{k}}=\frac{1}{\sqrt{N_{p}}} \sum_{\mathbf{R}_{p}, \alpha, i} \sqrt{m_{\alpha}} u_{p \alpha ; i} e^{-i \mathbf{k} \cdot \mathbf{R}_{p}} w_{-\mathbf{k} n ; i \alpha} .
\end{gathered}
$$

Here, $w_{\mathbf{k} n ; i \alpha}$ are the eigenvectors of the dynamical matrix and $n$ is the phonon branch index. In terms of phonon coordinates, which can always be chosen to be real, ${ }^{3}$ the vibrational Hamiltonian is

$$
\hat{H}_{\mathrm{vib}}=\sum_{n, \mathbf{k}}-\frac{1}{2} \frac{\partial^{2}}{\partial q_{n \mathbf{k}}^{2}}+\frac{1}{2} \omega_{n \mathbf{k}}^{2} q_{n \mathbf{k}}^{2},
$$

where $\omega_{n \mathbf{k}}^{2}$ are the eigenvalues of the dynamical matrix. This Hamiltonian consists of a sum of terms for noninteracting simple harmonic oscillators of frequencies $\omega_{n \mathbf{k}}$.

\section{Anharmonic approximation}

\section{Principal axes approximation to the $\mathrm{BO}$ energy surface}

To study anharmonic properties it is necessary to find an approximation to the BO energy surface that goes beyond the HA. The HA is often very accurate, and in such cases anharmonicity is expected to be a perturbation about the harmonic case. We write the BO energy surface as ${ }^{24}$

$$
\begin{aligned}
E_{\mathrm{el}}(\mathbf{Q})= & E_{\mathrm{el}}(\mathbf{0})+\sum_{n, \mathbf{k}} V_{n \mathbf{k}}\left(q_{n \mathbf{k}}\right) \\
& +\frac{1}{2} \sum_{n, \mathbf{k}} \sum_{n^{\prime}, \mathbf{k}^{\prime}} V_{n \mathbf{k} ; n^{\prime} \mathbf{k}^{\prime}}\left(q_{n \mathbf{k}}, q_{n^{\prime} \mathbf{k}^{\prime}}\right)+\cdots,
\end{aligned}
$$

where $\mathbf{Q}$ is a collective phonon vector with elements $q_{n \mathbf{k}}$, the primed sum indicates that the term $(n, \mathbf{k})=\left(n^{\prime}, \mathbf{k}^{\prime}\right)$ is 


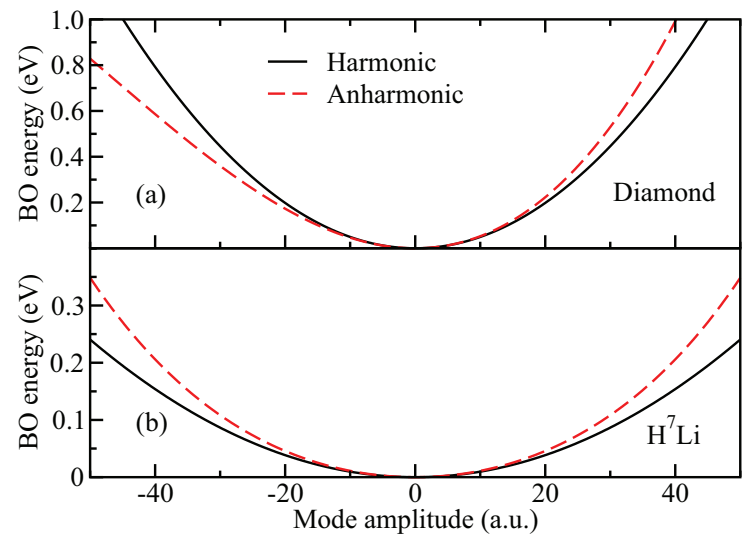

FIG. 1. (Color online) Harmonic and anharmonic BO energy surfaces per unit cell for an optical $\Gamma$-point phonon in (a) diamond and (b) $\mathrm{H}^{7} \mathrm{Li}$. The leading anharmonic term for diamond is cubic, and that for $\mathrm{H}^{7} \mathrm{Li}$ is quartic. For a harmonic ground-state wave function (Gaussian), the one-sigma amplitude is 9.1 a.u. for diamond and 13.7 a.u. for $\mathrm{H}^{7} \mathrm{Li}$. In real space, the one-sigma amplitudes correspond to 0.043 a.u. for diamond and 0.299 a.u. for $\mathrm{H}^{7} \mathrm{Li}$.

excluded, and the factor $1 / 2$ accounts for double counting. The independent phonon term takes the form

$$
V_{n \mathbf{k}}\left(q_{n \mathbf{k}}\right)=E_{\mathrm{el}}\left(0, \ldots, q_{n \mathbf{k}}, \ldots, 0\right)-E_{\mathrm{el}}(\mathbf{0}),
$$

but note that we do not assume the HA. The two-body term which introduces coupling between the modes takes the form

$$
\begin{aligned}
V_{n \mathbf{k} ; n^{\prime} \mathbf{k}^{\prime}}\left(q_{n \mathbf{k}}, q_{n^{\prime} \mathbf{k}^{\prime}}\right)= & E_{\mathrm{el}}\left(0, \ldots, q_{n \mathbf{k}}, \ldots, q_{n^{\prime} \mathbf{k}^{\prime}}, \ldots, 0\right) \\
& -V_{n \mathbf{k}}\left(q_{n \mathbf{k}}\right)-V_{n^{\prime} \mathbf{k}^{\prime}}\left(q_{n^{\prime} \mathbf{k}^{\prime}}\right)-E_{\mathrm{el}}(\mathbf{0}) .
\end{aligned}
$$

It is possible to continue this series for more general $N$-body terms, but because we start from the HA in which phonons are exactly noninteracting, it is expected that the higher order terms will decrease in magnitude as $N$ increases. [We note that Eq. (14) of Ref. 24 contains a typographical error connected with the coupling of vibrational modes.]

We show examples of the BO energy surface from independent phonon terms in Fig. 1 for diamond and lithium hydride. Both plots show the BO energy surface as a function of phonon amplitude for an optical phonon at the center of the Brillouin zone. The potential for diamond is asymmetric and the dominant anharmonic term is cubic. Diamond has a face-centered cubic (fcc) crystal structure with a two-atom basis at $(0,0,0)$ and $(a / 4, a / 4, a / 4)$, and a conventional cubic cell of lattice parameter $a$. The optical phonon represents the motion of the two atoms of the basis in opposite directions, leading to a steep potential when they approach, and a shallower potential when they move apart. The lithium hydride crystal structure is also fcc with a two-atom basis, but the second atom is located at $(a / 2, a / 2, a / 2)$, leading to a rather isotropic environment and a symmetric anharmonic potential. In this case the anharmonicity arises because the potential is steeper than the harmonic one, and the leading anharmonic term is quartic.

The crystal structure determines the symmetry operations relating different $\mathbf{k}$ points within the first Brillouin zone. This implies that the only points that need to be treated explicitly are those within the irreducible wedge of the Brillouin zone, which reduces the computational cost significantly. For example, a $5 \times 5 \times 5$ supercell of the diamond structure has $125 \mathbf{k}$ points, of which only 22 need be treated explicitly.

\section{Vibrational self-consistent field}

Within the principal axes approximation to the BO energy surface the vibrational Hamiltonian is

$$
\hat{H}_{\mathrm{vib}}=\sum_{n, \mathbf{k}}-\frac{1}{2} \frac{\partial^{2}}{\partial q_{n \mathbf{k}}^{2}}+E_{\mathrm{el}}(\mathbf{Q}, \beta),
$$

and the Schrödinger equation is solved by an anharmonic vibrational wave function $|\Phi(\mathbf{Q})\rangle$ and corresponding anharmonic energy $E_{\text {vib }}$, where $\hat{H}_{\text {vib }}|\Phi(\mathbf{Q})\rangle=E_{\text {vib }}|\Phi(\mathbf{Q})\rangle$. In Eq. (10), $\beta$ is the inverse temperature. In principle it would be straightforward to calculate the BO energy surface with electrons distributed, for example, according to the Fermi-Dirac distribution at some finite temperature without substantial additional cost. This temperature dependence can be important for metallic systems, but we will drop it in the rest of this paper, because here we only study systems with a band gap.

To solve this equation, the energy is minimized with respect to a set of single-phonon states $\left\{\left|\phi_{n \mathbf{k}}\left(q_{n \mathbf{k}}\right)\right\rangle\right\}$ that form a Hartree product for the trial wave function $|\Phi(\mathbf{Q})\rangle=\prod_{n, \mathbf{k}}\left|\phi_{n \mathbf{k}}\left(q_{n \mathbf{k}}\right)\right\rangle$. This leads to the vibrational self-consistent field equations ${ }^{22}$

$$
\left(-\frac{1}{2} \frac{\partial^{2}}{\partial q_{n \mathbf{k}}^{2}}+\bar{V}_{n \mathbf{k}}\left(q_{n \mathbf{k}}\right)\right)\left|\phi_{n \mathbf{k}}\left(q_{n \mathbf{k}}\right)\right\rangle=\lambda_{n \mathbf{k}}\left|\phi_{n \mathbf{k}}\left(q_{n \mathbf{k}}\right)\right\rangle,
$$

where $\lambda_{n \mathbf{k}}$ is a vibrational energy eigenvalue and

$$
\bar{V}_{n \mathbf{k}}\left(q_{n \mathbf{k}}\right)=\left\langle\prod_{n^{\prime}, \mathbf{k}^{\prime}}^{\prime} \phi_{n^{\prime} \mathbf{k}^{\prime}}\left(q_{n^{\prime} \mathbf{k}^{\prime}}\right)\left|E_{\mathrm{el}}(\mathbf{Q})\right| \prod_{n^{\prime}, \mathbf{k}^{\prime}}^{\prime} \phi_{n^{\prime} \mathbf{k}^{\prime}}\left(q_{n^{\prime} \mathbf{k}^{\prime}}\right)\right\rangle .
$$

The primed products indicate that the term $(n, \mathbf{k})$ is excluded. The final energy is

$$
\begin{aligned}
E_{\mathrm{vib}}= & \sum_{n, \mathbf{k}} \lambda_{n \mathbf{k}}+\left\langle\prod_{n, \mathbf{k}} \phi_{n \mathbf{k}}\left(q_{n \mathbf{k}}\right)\right| E_{\mathrm{el}}(\mathbf{Q}) \\
& -\sum_{n, \mathbf{k}} \bar{V}_{n \mathbf{k}}\left(q_{n \mathbf{k}}\right)\left|\prod_{n, \mathbf{k}} \phi_{n \mathbf{k}}\left(q_{n \mathbf{k}}\right)\right\rangle .
\end{aligned}
$$

The VSCF equations determine a set of excited eigenstates for each degree of freedom. These can be used to construct approximate anharmonic excited state wave functions as

$$
\left|\Phi^{\mathbf{S}}(\mathbf{Q})\right\rangle=\prod_{n, \mathbf{k}}\left|\phi_{n \mathbf{k}}^{S_{n \mathbf{k}}}\left(q_{n \mathbf{k}}\right)\right\rangle,
$$

where the superindices $S_{n \mathbf{k}}$ label the excited states, and $\mathbf{S}$ is a vector with elements $S_{n \mathbf{k}}$. The corresponding energies are labeled by $E_{\mathbf{S}}$.

A perturbation theory can be constructed on the VSCF equations, ${ }^{23}$ and the second-order correction to the energy of state $\mathbf{S}$ is

$$
\begin{aligned}
E_{\mathrm{vib}, \mathbf{S}}^{(2)}= & \sum_{\mathbf{S}^{\prime} \neq \mathbf{S}} \frac{1}{E_{\mathbf{S}}-E_{\mathbf{S}^{\prime}}} \\
& \times\left|\left\langle\prod_{n, \mathbf{k}} \phi_{n \mathbf{k}}^{S_{n \mathbf{k}}^{\prime}}\left|E_{\mathrm{el}}(\mathbf{Q})-\sum_{n, \mathbf{k}} \bar{V}_{n \mathbf{k}}\left(q_{n \mathbf{k}}\right)\right| \prod_{n, \mathbf{k}} \phi_{n \mathbf{k}}^{S_{n \mathbf{k}}}\right\rangle\right|^{2} .
\end{aligned}
$$


The anharmonic free energy $F$ can be calculated at any inverse temperature $\beta$ from the eigenvalues that solve the VSCF equations as

$$
F=-\frac{1}{\beta} \ln \mathcal{Z}
$$

where $\mathcal{Z}=\sum_{\mathrm{S}} e^{-\beta E_{\mathrm{S}}}$ is the partition function.

\section{PHONON EXPECTATION VALUES}

\section{A. General formulation}

The wave function $|\Phi(\mathbf{Q})\rangle$ that solves the VSCF equations contains, in principle, all of the physical information about the vibrational system within the principal axes approximation. For an operator $\hat{O}(\mathbf{Q})$ that depends on the phonon coordinates, it is then possible to evaluate the expectation value with respect to the phonon wave function as

$$
\langle\hat{O}(\mathbf{Q})\rangle_{\Phi}=\langle\Phi(\mathbf{Q})|\hat{O}(\mathbf{Q})| \Phi(\mathbf{Q})\rangle .
$$

The expansion of the operator $\hat{O}(\mathbf{Q})$ in terms of the phonon amplitudes can be written in analogy to the expression for the BO energy surface as

$$
\begin{aligned}
\hat{O}(\mathbf{Q})= & \hat{O}(\mathbf{0})+\sum_{n, \mathbf{k}} \hat{O}_{n \mathbf{k}}\left(q_{n \mathbf{k}}\right) \\
& +\sum_{n, \mathbf{k}} \sum_{n^{\prime}, \mathbf{k}^{\prime}} \hat{O}_{n \mathbf{k} ; n^{\prime} \mathbf{k}^{\prime}}\left(q_{n \mathbf{k}}, q_{n^{\prime} \mathbf{k}^{\prime}}\right)+\cdots
\end{aligned}
$$

This expression can be constructed from data accumulated during the mapping of the BO energy surface along the principal axes.

Within the finite-temperature formalism, the expectation value of the operator $\hat{O}(\mathbf{Q})$ at inverse temperature $\beta$ is

$$
\langle\hat{O}(\mathbf{Q})\rangle_{\Phi, \beta}=\frac{1}{\mathcal{Z}} \sum_{\mathbf{S}}\left\langle\Phi^{\mathbf{S}}(\mathbf{Q})|\hat{O}(\mathbf{Q})| \Phi^{\mathbf{S}}(\mathbf{Q})\right\rangle e^{-\beta E_{\mathbf{S}}} .
$$

Examples of phonon-dependent operators are electronic eigenvalues, the stress tensor, and the atomic positions. In this paper we will describe electronic eigenvalues and their relation to electron-phonon interactions and the stress tensor and its relation to thermal expansion.

\section{B. Electron-phonon interactions}

The effects of electron-phonon interactions on the electronic band structure of solids can be calculated by renormalizing the phonons due to interactions with the electronic states $^{26,27}$ or renormalizing the electrons due to their interactions with the phonons. ${ }^{18}$ These two approaches can be shown to be equivalent by Brooks' theorem. ${ }^{28}$

We accumulate the single-particle electronic band structure while mapping the $\mathrm{BO}$ energy surface within the principal axes approximation. Using the expansion above for the phonon amplitude dependence, it is then possible to calculate the renormalized electronic band structure due to the presence of the phonons.

\section{Stress tensor}

The differential Gibbs free energy $d G$ of a system with an externally applied stress $\sigma_{i j}^{\text {ext }}$ is ${ }^{29}$

$$
d G=d F_{\mathrm{el}}+d F_{\mathrm{vib}}-\Omega \sum_{i, j} \sigma_{i j}^{\mathrm{ext}} d \epsilon_{i j},
$$

where $\epsilon_{i j}$ is the strain tensor and $\Omega$ is the volume. The internal vibrational stress tensor is defined as

$$
\sigma_{i j}^{\mathrm{vib}}=-\frac{1}{\Omega} \frac{\partial F_{\mathrm{vib}}}{\partial \epsilon_{i j}} .
$$

The vibrational energy is an approximately linear function of volume for the configurations of interest in thermal expansion. This leads to a constant vibrational stress tensor. It is then possible to define an effective stress $\sigma_{i j}^{\text {eff }}=\sigma_{i j}^{\text {ext }}+\sigma_{i j}^{\text {vib }}$ such that Eq. (20) can be rewritten as

$$
d G=d F_{\mathrm{el}}-\Omega \sum_{i, j} \sigma_{i j}^{\mathrm{eff}} d \epsilon_{i j}
$$

The minimum of the Gibbs free energy with respect to variations in strain corresponds to the equilibrium configuration of the system. Therefore, minimizing the Gibbs free energy in Eq. (22), where the vibrational dependence is implicit in the effective stress $\sigma_{i j}^{\text {eff }}$, leads to the equilibrium configuration for the full vibrational system. If the vibrational stress varies significantly in the region of interest, an iterative approach can be adopted. Equation (22) can be used to determine a new equilibrium configuration, which can be used as a starting point for the next calculation. This iterative process can be repeated until convergence is reached. In this work, only one or two iterations were required for convergence.

The stress tensor arising from the electronic Hamiltonian can be calculated for each point sampled on the BO energy surface, and its expectation value with respect to the phonon wave function evaluated using Eq. (19). This stress, $\sigma_{i j}^{\mathrm{vib}, \mathrm{V}}$, is the contribution of the potential energy to the vibrational stress. In general, the internal stress tensor has contributions from both the kinetic and potential parts of the Hamiltonian. The contribution from the kinetic part, ${ }^{30}$

$$
\sigma_{i j}^{\mathrm{vib}, \mathrm{T}}=-\frac{1}{\Omega}\left\langle\Phi\left|\sum_{\mathbf{R}_{p}, \alpha} m_{\alpha} \dot{u}_{p \alpha ; i} \dot{u}_{p \alpha ; j}\right| \Phi\right\rangle,
$$

is therefore also required. The symbol $\dot{u}$ indicates the time derivative of a displacement coordinate. For an isotropic system where the internal vibrational stress can be described by a pressure $P$, the contribution from the kinetic part becomes $3 P=-\operatorname{tr} \sigma^{\mathrm{vib}, \mathrm{T}}=2 E_{\mathrm{vib}}^{\mathrm{T}} / \Omega$, where tr denotes the trace of the tensor and $E_{\mathrm{vib}}^{\mathrm{T}}$ is the vibrational kinetic energy.

The total vibrational stress $\sigma_{i j}^{\mathrm{vib}}=\sigma_{i j}^{\mathrm{vib}, \mathrm{V}}+\sigma_{i j}^{\mathrm{vib}, \mathrm{T}}$ can then be used in tandem with Eq. (22) to determine the equilibrium configuration in the presence of vibrations and at finite temperature.

\section{COMPUTATIONAL DETAILS}

\section{A. Electronic calculations}

We have solved the electronic Schrödinger equation within plane-wave DFT $^{31,32}$ using ultrasoft pseudopotentials ${ }^{33}$ as 
implemented in the CASTEP code. ${ }^{34}$ All energy differences were converged to within $10^{-4} \mathrm{eV}$ per unit cell and all stresses were converged to within $10^{-2} \mathrm{GPa}$. This level of convergence required an energy cutoff of $1000 \mathrm{eV}$ and a reciprocal-space Monkhorst-Pack ${ }^{35}$ grid of spacing $2 \pi \times$ $0.04 \AA^{-1}$. We have used the local density approximation (LDA $)^{36,37}$ to the exchange-correlation functional for the diamond calculations, and the Perdew-Burke-Ernzerhof $(\mathrm{PBE})^{38}$ generalized gradient approximation density functional for the lithium hydride and deuteride calculations.

\section{B. Vibrational calculations}

We have mapped the BO energy surface along the phonon modes with a maximum amplitude given by a multiple (between 3 and 5) of the harmonic expectation value of the mode amplitude

$$
\left\langle q_{n \mathbf{k}}^{2}\right\rangle=\frac{1}{\omega_{n \mathbf{k}}}\left(\frac{1}{2}+\frac{1}{e^{\beta \omega_{n \mathbf{k}}}-1}\right) .
$$

We have fitted the independent phonon term with a polynomial of order 6 , and tests with polynomials of order up to 8 have confirmed the convergence of the results. For the twobody terms we have fitted the two-parameter functional form $V_{n \mathbf{k} ; n^{\prime} \mathbf{k}^{\prime}}=c_{n \mathbf{k} ; n^{\prime} \mathbf{k}^{\prime}}^{(1)} q_{n \mathbf{k}} q_{n^{\prime} \mathbf{k}^{\prime}}+c_{n \mathbf{k} ; n^{\prime} \mathbf{k}^{\prime}}^{(2)} q_{n \mathbf{k}}^{2} q_{n^{\prime} \mathbf{k}^{\prime}}^{2}$. The first term is the lowest order coupling term in a Taylor expansion of the potential. The second term is not the next order term, but the lowest order term that gives a nonzero contribution when overlapped with the harmonic ground state, and, for this reason, it is expected to be larger than other lower order cross-terms. Numerical tests have confirmed this.

We use simple harmonic oscillator eigenstates as a basis set for expanding the anharmonic wave function in the VSCF equations. The simple harmonic oscillator frequencies used are those of a quadratic fit to the anharmonic independent-phonon BO energy surface. We have used basis sets including 100 states for each mode, checking the convergence of the results by using larger sets.

\section{RESULTS}

\section{A. Anharmonic energy}

The mapping of the BO energy surface within the principal axes approximation requires the displacement of the atomic nuclei inside a periodic supercell. The supercell size determines a set of commensurate reciprocal-space points associated with the harmonic phonon modes. Convergence with respect to supercell size must be tested.

The sampling of the Brillouin zone within the HA is not constrained by the supercell size in the same manner. This is because within the HA, all that is needed is the dynamical matrix at the $\mathbf{k}$ points of interest, and this matrix can be constructed at general $\mathbf{k}$ points irrespective of the supercell size. This is accomplished by first constructing the real-space matrix of force constants and then Fourier transforming to the dynamical matrix at a general $\mathbf{k}$ point. This approach relies on the fact that the force constants tend rapidly to zero as the separation between atoms increases. The matrix of force constants can be constructed using a finite-displacement method $^{39}$ or density functional perturbation theory ${ }^{40,41}$ to

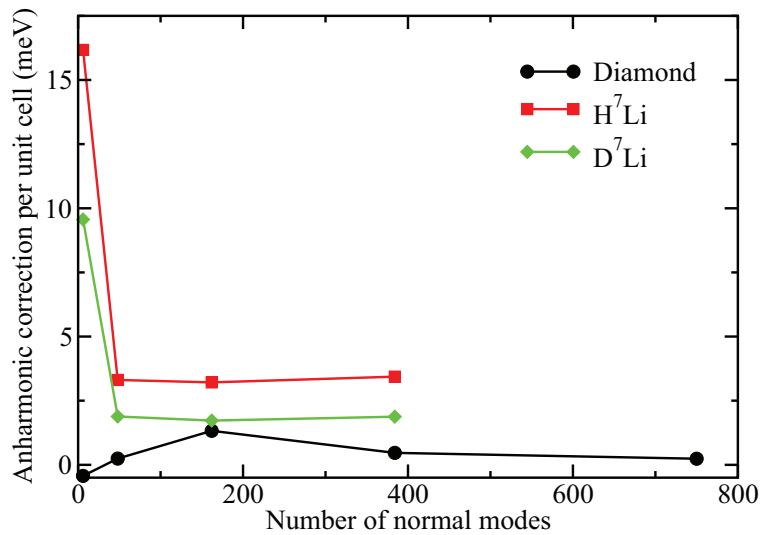

FIG. 2. (Color online) Anharmonic energy correction as a function of the number of normal modes (supercell size) for diamond, $\mathrm{H}^{7} \mathrm{Li}$, and $\mathrm{D}^{7} \mathrm{Li}$. The anharmonic correction is larger for the lighter elements.

evaluate the dynamical matrices on a coarse k-point grid, and then calculating the matrix of force constants by means of an inverse Fourier transform.

The strategy we follow is to calculate both the harmonic $F_{\text {har }}$ and anharmonic $F_{\text {anh }}$ free energies from the $\mathbf{k}$ points commensurate with the supercell. The anharmonic correction is then evaluated as $\Delta F_{\text {anh }}=F_{\text {anh }}-F_{\text {har }}$, and converged with respect to supercell size. This correction is then added to an independently converged harmonic free energy to give the total anharmonic energy.

The supercell-size dependence of $\Delta F_{\text {anh }}$ at zero temperature for diamond, lithium hydride, and lithium deuteride is shown in Fig. 2. The anharmonic energy is evaluated using only the independent phonon terms in Eq. (8). The energy difference between the last two successive points in each curve is smaller than $0.2 \mathrm{meV}$ per unit cell. Table I gives the numerical values of the harmonic phonon energy and the independent phonon anharmonic correction per unit cell.

The anharmonicity of diamond is small. For the smallest supercell size corresponding to the $\Gamma$ point only, the anharmonic correction is negative, as expected for cubic anharmonicity ${ }^{42}$ (see Fig. 1). Including more $\mathbf{k}$ points reverses the sign of the anharmonic correction, but it remains small.

The different isotopic masses of $\mathrm{H}^{7} \mathrm{Li}$ and $\mathrm{D}^{7} \mathrm{Li}$ lead to different vibrational properties. The anharmonic correction is larger for $\mathrm{H}^{7} \mathrm{Li}$ than for $\mathrm{D}^{7} \mathrm{Li}$ because the lighter elements explore wider regions of the $\mathrm{BO}$ energy surface and encounter more anharmonicity. We have also performed calculations with the lithium isotope ${ }^{6} \mathrm{Li}$, but the results are not much affected by

TABLE I. Converged anharmonic free energy correction per unit cell at zero temperature using independent phonons for the mapping of the BO energy surface along the principal axes. The converged harmonic energy is also displayed.

\begin{tabular}{lccc}
\hline \hline & Num. modes & $F_{\text {har }}(\mathrm{meV})$ & $\Delta F_{\text {anh }}(\mathrm{meV})$ \\
\hline Diamond & 750 & 367.59 & 0.23 \\
$\mathrm{H}^{7} \mathrm{Li}$ & 384 & 221.49 & 3.21 \\
$\mathrm{D}^{7} \mathrm{Li}$ & 384 & 174.75 & 1.88 \\
\hline \hline
\end{tabular}






FIG. 3. (Color online) Anharmonic two-dimensional BO energy surface per unit cell for a pair $\left(q_{1}, q_{2}\right)$ of optical modes at the $\Gamma$ point for $\mathrm{H}^{7} \mathrm{Li}$. We plot (a) the exact energy surface and (b) the approximate energy surface for independent phonons only. For a harmonic groundstate wave function (Gaussian), the one-sigma amplitude is 13.7 a.u.

this isotopic substitution because it represents a mass reduction of only about $15 \%$, compared to the doubling of mass from substituting hydrogen by deuterium.

We have studied the effects of including the phonon coupling term in Eq. (9) for a $2 \times 2 \times 2$ supercell ( 48 modes) of $\mathrm{H}^{7} \mathrm{Li}$. The anharmonic coupling correction has the opposite effect to the independent phonon correction, and the final anharmonic correction with coupling between modes is $\Delta F_{\text {anh }}=+0.79 \mathrm{meV}$, compared to $\Delta F_{\text {anh }}=+3.21 \mathrm{meV}$ with independent phonons only (see Table I). Using the second-order perturbation theory of Eq. (15) does not change the final result appreciably, indicating that the energies have converged with respect to the mean-field theory. This means that, like diamond, $\mathrm{H}^{7} \mathrm{Li}$ has a small anharmonic energy correction, but, unlike diamond, the reason for this is the cancellation of the contributions from independent phonons and pairwise phonon coupling. This negative contribution from the coupling terms can be better appreciated by referring to Fig. 3. We show the coupling BO energy surface $V_{1 ; 2}\left(q_{1}, q_{2}\right)$ for two optical phonons $\left(q_{1}, q_{2}\right)$ at the $\Gamma$ point compared to the approximate surface constructed from the independent phonon terms only. The accurate potential is shallower than the approximate one, leading to the smaller anharmonic correction when including phonon coupling. The corresponding harmonic two-dimensional subspace is very similar to the approximate (bottom) potential in Fig. 3, but with concentric circular equipotential lines because the harmonic phonon frequencies are degenerate in the case shown.

Nolan and co-workers ${ }^{43}$ have used very accurate electronic structure methods to calculate the cohesive energy of lithium hydride, including the zero point (ZP) energy at the harmonic level. Their accuracy is of the same order of magnitude as the anharmonic corrections we have calculated for this system.

Figure 2 shows that convergence with respect to supercell size is reached with smaller cells for $\mathrm{H}^{7} \mathrm{Li}$ and $\mathrm{D}^{7} \mathrm{Li}$ than for diamond. In diamond, the phonons with the largest anharmonic contribution to the energy are located in the third phonon branch, corresponding to the highest energy acoustic modes. These modes represent large-scale atomic displacements and larger supercells are needed to describe them. In contrast, the largest anharmonic contributions in $\mathrm{H}^{7} \mathrm{Li}$ and $\mathrm{D}^{7} \mathrm{Li}$ arise from the optical branches, which represent small-scale displacements of the atoms within the primitive cells. This local anharmonic behavior is reflected by convergence at smaller supercell sizes.

\section{B. Band gap renormalization}

We have used the phonon expectation value formalism to investigate the $\mathrm{ZP}$ renormalization and temperature dependence of band gaps in diamond and isotopes of lithium hydride. In both cases we have selected the thermal band gap (smallest band gap), which is indirect for diamond and direct for the lithium hydride systems. For diamond, the top of the valence band is at the electronic $\Gamma$ point, and the bottom of the conduction band is along the line joining the $\Gamma$ and $X$ points. The thermal gap is at the electronic $X$ point for the lithium hydride isotopes. In all calculations reported in this section the anharmonicity has been treated at the independent phonon level.

We show the temperature dependence of the electronic thermal band gap $E_{\mathrm{g}}$ of diamond in Fig. 4. The black diamonds are experimental results from Ref. 44. The ZP band gap renormalization is $E_{\mathrm{g}}^{\mathrm{ZP}}=-462 \mathrm{meV}$. This value is $92 \mathrm{meV}$ larger than that estimated from the asymptotic behavior at high temperature in Ref. 45 . The analysis in Ref. 45 suffers from

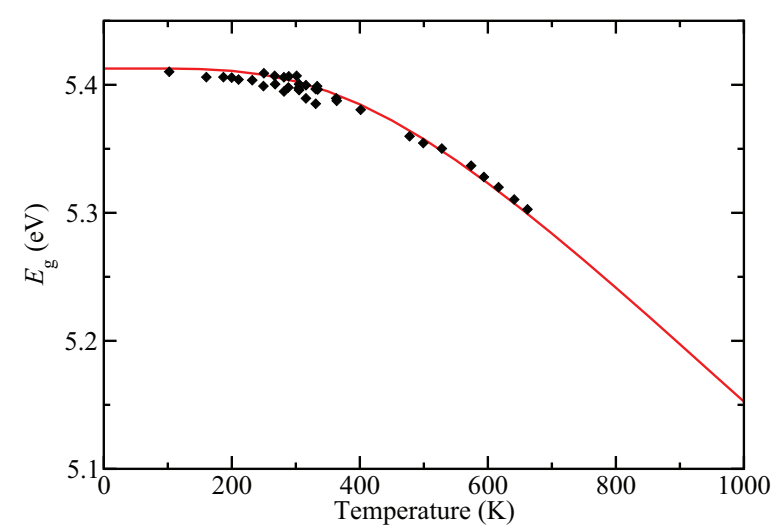

FIG. 4. (Color online) Temperature dependence of the thermal band gap $E_{\mathrm{g}}$ of diamond. The DFT result (red solid curve) is offset to match experimental data (black diamonds) at zero temperature. The experimental data are from Ref. 44. 


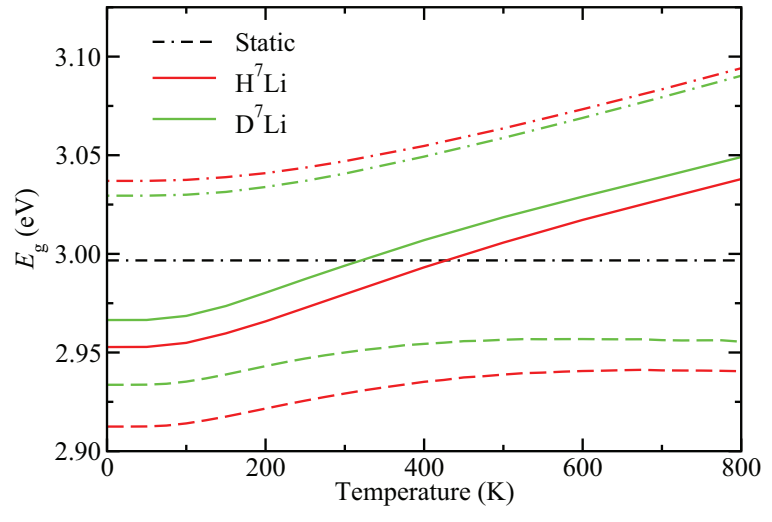

FIG. 5. (Color online) Temperature dependence of the thermal band gap $E_{\mathrm{g}}$ of lithium hydride for the isotopes $\mathrm{H}^{7} \mathrm{Li}$ (red) and $\mathrm{D}^{7} \mathrm{Li}$ (green) calculated within DFT. The black double-dashed-dotted line indicates the value of the static band gap. Dashed lines refer to the renormalized gaps due to electron-phonon interactions, dashed-dotted lines refer to the renormalization due to thermal expansion with temperature, and the solid lines are the sum of the two contributions.

the lack of experimental data beyond the Debye temperature, where the asymptotic behavior should occur, and an analytic functional form with the correct asymptotic behavior was fitted to the data instead. We attribute some of the discrepancy to the inaccuracy of the fitted function used in Ref. 45. Our firstprinciples results can also be compared with other theoretical studies of the thermal gap of diamond ${ }^{46}$ based on the empirical pseudopotential method ${ }^{47}$ which find a ZP renormalization of approximately $-620 \mathrm{meV}$. The calculations reported in Ref. 46 lead to an overestimate of the reduction in band gap with temperature compared to experiment. This could explain the larger ZP renormalization found in Ref. 46 compared to our first-principles results. The band gap renormalization has an additional intrinsic contribution from the change in volume of the crystal with temperature. For diamond this contribution is very small, amounting to a decrease of $16 \mathrm{meV}$ for the temperature range in Fig. 4.

Figure 5 shows the temperature dependence of the thermal band gaps of $\mathrm{H}^{7} \mathrm{Li}$ and $\mathrm{D}^{7} \mathrm{Li}$. The $\mathrm{ZP}$ renormalizations arising from electron-phonon interactions are $E_{\mathrm{g}}^{\mathrm{ZP}}=-84 \mathrm{meV}$ and $E_{\mathrm{g}}^{\mathrm{ZP}}=-62 \mathrm{meV}$, respectively, and those arising from the change in volume with temperature are $E_{\mathrm{g}}^{\mathrm{ZP}}=+40 \mathrm{meV}$ and $E_{\mathrm{g}}^{\mathrm{ZP}}=+33 \mathrm{meV}$, respectively. The isotope effect for the lighter compound leads to a larger band gap renormalization for both electron-phonon and volume contributions. This is in agreement with the expectation that lighter isotopes show larger atomic displacements.

It is interesting to note in the lithium hydride systems that the ZP electron-phonon renormalizations are negative (gap closing), while opening of the gap is observed at finite temperature. To investigate this we have calculated the contribution to the electron-phonon renormalization from each phonon mode, and the results indicate that low-energy phonons tend to open the gap whereas high-energy phonons tend to close it. At the ZP level, all phonons contribute, and the high-energy gap-closing phonons dominate. However, as the temperature is increased, the lower energy phonons are excited more easily, leading to opening of the gap. At higher temperatures, when the high-energy phonons are excited as well, the trend is expected to revert to gap closing. In Fig. 5, the higher temperatures explored (close to the melting point) only show a leveling off of the electron-phonon correction. Extending the calculations to higher unphysical temperatures (not shown) does take the system into the gap-closing regime as expected.

Our first-principles calculations for lithium hydride are, as far as we are aware, the first to show nonmonotonic behavior in the temperature dependence of a band gap due to electron-phonon interactions. This behavior has been observed experimentally in ternary semiconductors ${ }^{48,49}$ and it has been described by an analytic functional form consisting of the sum of two Bose-Einstein oscillators of opposite sign and with energies related to the low and high frequency phonon modes. ${ }^{49}$ Our calculations show that this difference in behavior between the low and high frequency phonons is responsible for the nonmonotonic variation of the gap with temperature.

In Fig. 5 we also show the sum of the contributions to the gap renormalization from electron-phonon interactions and thermal expansion for $\mathrm{H}^{7} \mathrm{Li}$ and $\mathrm{D}^{7} \mathrm{Li}$. This simple addition of the two terms is the standard procedure used in the literature. ${ }^{19}$ However, this approximation might not be very accurate for a system such as lithium hydride with a large thermal expansion (see Sec. VC below). We calculated the electron-phonon interaction renormalization to the band gap at the volume including $\mathrm{ZP}$ motion for $\mathrm{H}^{7} \mathrm{Li}$, and the real renormalization is $4 \mathrm{meV}$ larger than the one found by the simple addition procedure.

In Fig. 4, a constant energy shift has been added to the theoretical curve to match experiment at zero temperature. Standard approximations to the DFT exchange-correlation functional such as the LDA or PBE do not reproduce the derivative discontinuity with respect to particle number of the exact functional. This leads to a severe underestimation of band gaps in semiconductors and insulators. ${ }^{50,51}$ This problem can be addressed in various ways, the most straightforward being the application of a scissor operator ${ }^{50,52}$ to the band gaps. When calculating band-gap renormalization, we are interested in the change in the band gap, rather than its absolute value. The fact that all atomic configurations suffer from a similar gap underestimation suggests that the calculated changes may be accurate even when using standard functionals. This is supported by the calculations of Giustino and co-workers ${ }^{20}$ of the electron-phonon band-gap renormalization in diamond using Allen-Heine-Cardona perturbation theory. ${ }^{18,19}$ They use both the LDA and the LDA corrected with a scissor operator, obtaining consistent results. Note that Giustino and co-workers look at the direct optical gap instead of the thermal gap that we have studied. Cannuccia and Marini ${ }^{53,54}$ use many-body perturbation theory to study the optical gap of diamond as well, and they report a significant structure in the spectral function beyond the quasiparticle picture, corresponding to subgap polaronic states.

\section{Thermal expansion}

We have used the formalism described in Sec. IIIC to calculate the internal vibrational stress and the corresponding equilibrium configuration of the crystal as a function of 


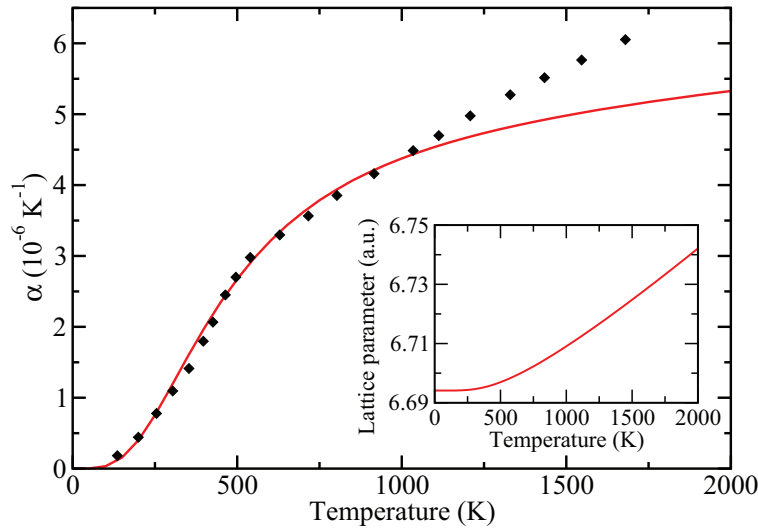

FIG. 6. (Color online) Temperature dependence of the coefficient of linear expansion $\alpha$ for diamond. The black diamonds are experimental results from Ref. 55. The inset shows the temperature dependence of the lattice parameter.

temperature. The structures of diamond, $\mathrm{H}^{7} \mathrm{Li}$, and $\mathrm{D}^{7} \mathrm{Li}$ are described by a single parameter, so the different equilibrium configurations differ only in the volume. We present results for the thermal expansion of diamond, $\mathrm{H}^{7} \mathrm{Li}$, and $\mathrm{D}^{7} \mathrm{Li}$. All calculations reported in this section have treated anharmonicity at the independent-phonon level.

In Fig. 6 we show the temperature dependence of the lattice parameter $a(T)$ of diamond and the corresponding temperature dependence of the linear coefficient of thermal expansion,

$$
\alpha(T)=\frac{1}{a} \frac{d a}{d T} .
$$

We compare our results with experimental data from Ref. 55 . The agreement is good for temperatures below $1200 \mathrm{~K}$, but at higher temperatures the calculated $\alpha(T)$ underestimates the experimental values. This effect is also seen in calculations using the quasiharmonic approximation. ${ }^{15}$ Our methodology assumes a volume-independent internal vibrational stress tensor for the volumes of interest at each iteration, as described in Sec. III C. Iterating the calculation at the volume including the ZP phonon pressure leads to a change in the phonon pressure of diamond of less than $-0.05 \mathrm{GPa}$, which represents

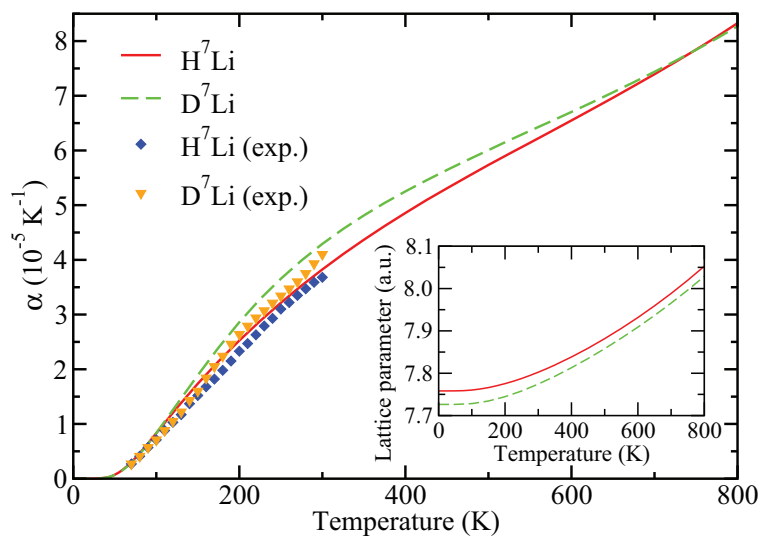

FIG. 7. (Color online) Temperature dependence of the coefficient of linear expansion $\alpha$ for lithium hydride with isotopic compositions $\mathrm{H}^{7} \mathrm{Li}$ and $\mathrm{D}^{7} \mathrm{Li}$. The experimental data are from Ref. 56. The inset shows the temperature dependence of the lattice parameters.
TABLE II. Static DFT and ZP renormalized lattice parameters of diamond, $\mathrm{H}^{7} \mathrm{Li}$, and $\mathrm{D}^{7} \mathrm{Li}$.

\begin{tabular}{lccc}
\hline \hline & $a^{\text {static }}($ a.u. $)$ & $a^{\mathrm{ZP}}($ a.u. $)$ & $\Delta a$ (a.u.) \\
\hline Diamond (LDA) & 6.669 & 6.694 & +0.025 \\
$\mathrm{H}^{7} \mathrm{Li}(\mathrm{PBE})$ & 7.600 & 7.758 & +0.158 \\
$\mathrm{D}^{7} \mathrm{Li}(\mathrm{PBE})$ & 7.600 & 7.726 & +0.126 \\
\hline \hline
\end{tabular}

less than $1.5 \%$ of the total phonon pressure. This means that for diamond a single iteration is sufficient for obtaining converged results.

The results for the lithium hydride isotopes are shown in Fig. 7 and are compared with experimental data from Ref. 56. The lattice parameters of the different isotopes show the expected behavior, with the lighter compound leading to a larger $\mathrm{ZP}$ phonon pressure and therefore to a larger lattice expansion. In contrast, the linear coefficient of thermal expansion is larger for the heavier deuterium compound, in agreement with experiment. The phonons of $\mathrm{D}^{7} \mathrm{Li}$ have lower energies than those of $\mathrm{H}^{7} \mathrm{Li}$, and therefore they are excited more easily with increasing temperature. This leads to the thermal expansion coefficient being larger for $\mathrm{D}^{7} \mathrm{Li}$ at low temperatures.

The volume change in lithium hydride due to $\mathrm{ZP}$ motion is much larger than that in diamond. The change in ZP phonon pressure between the first and second iterations is as large as $-0.22 \mathrm{GPa}$, representing $11 \%$ of the absolute value. In this case the second iteration is important for obtaining accurate results. The change in pressure is negative because the expanded lattice (after the first iteration) has softer phonons than the static DFT lattice, which leads to smaller phonon pressures.

The absolute values of the lattice parameters within DFT are known to deviate systematically by a few percent from experimental results. However, the differences in lattice parameters within a given DFT approximation are expected to be accurate. These are displayed in Table II. Mounet and Marzari (Ref. 15) find the same ZP expansion for diamond using the PBE functional, rather than the LDA that we have used, even though the absolute value of the lattice parameter is somewhat different. Anderson and co-workers ${ }^{56}$ report experimental results for the lattice parameter of $\mathrm{H}^{7} \mathrm{Li}$ and $\mathrm{D}^{7} \mathrm{Li}$ at a temperature of $83 \mathrm{~K}$, finding a difference between the value for the two isotopes of 0.032 a.u., in good agreement with the value of 0.034 a.u. we have calculated.

\section{CONCLUSIONS}

We have described a formalism for studying temperaturedependent anharmonic vibrational properties in periodic systems using first-principles calculations. The BO energy surface is mapped using a principal axes approximation, and the resulting equations are solved using a Hartree mean-field approach and second-order perturbation theory. We then use the vibrational anharmonic wave function to calculate expectation values of phonon-dependent quantities. In particular, we have studied the effects of electron-phonon interactions on electronic band gaps, and the role of the stress tensor in thermal expansion. Other quantities can be studied within this 
framework, such as the mean atomic positions within the unit cell.

The methodology we have presented involves the solution of the electronic Schrödinger equation at a set of low-energy atomic configurations. Our results are based on density functional theory calculations, but the methodology is independent of the particular electronic structure method used. For instance, for larger systems it might be necessary to use force fields, or, if higher accuracy is required, quantum Monte Carlo methods. ${ }^{57}$

We have tested the formalism on diamond, lithium hydride, and lithium deuteride, which exhibit quite small anharmonicities. However, while diamond is nearly harmonic, the small anharmonicity in lithium hydride arises from a cancellation of the contributions from single-phonon and two-phonon terms. The results for both band-gap renormalization and lattice expansion as a function of temperature have been found to be in good agreement with experimental results for diamond.
For $\mathrm{H}^{7} \mathrm{Li}$ and $\mathrm{D}^{7} \mathrm{Li}$, our thermal expansion results also agree with experiment but, as far as we are aware, there are no experimental data for the temperature dependence of the band gap and our results serve as a prediction that could be tested.

In our calculations using the VSCF method plus perturbation theory we appear to have converged the results with respect to the description of anharmonicity. This suggests that our approach can be used to estimate anharmonic effects in systems with substantially stronger anharmonicity than diamond and lithium hydride.

\section{ACKNOWLEDGMENTS}

We thank the Engineering and Physical Sciences Research Council (UK) for financial support. The calculations were performed on the Cambridge High Performance Computing Service facility. *bm418@cam.ac.uk

${ }^{1}$ D. C. Wallace, Thermodynamics of Crystals (John Wiley \& Sons, New York, 1972).

${ }^{2}$ M. Born and K. Huang, Dynamical Theory of Crystal Lattices (Oxford University Press, Oxford, 1956).

${ }^{3}$ A. A. Maradudin, E. W. Montroll, G. H. Weiss, and I. P. Ipatova, Theory of Lattice Dynamics in the Harmonic Approximation, 2nd ed. (Academic Press, New York, 1971).

${ }^{4} \mathrm{M}$. Born, Festschrift zur Feier des Zweihundertjährigen Bestehens der Akademie der Wissenschaften der Universität Götttingen I. Mathematisch-Physikalische Klasse (Springer-Verlag, Berlin, 1951).

${ }^{5}$ D. Hooton, Z. Phys. 142, 42 (1955).

${ }^{6}$ M. Born and D. Hooton, Z. Phys. 142, 201 (1955).

${ }^{7}$ D. J. Hooton, Philos. Mag. 3, 49 (1958).

${ }^{8}$ P. Choquard, The Anharmonic Crystal (W. A. Benjamin Inc., New York, 1967).

${ }^{9}$ N. R. Werthamer, Phys. Rev. B 1, 572 (1970).

${ }^{10}$ M. Klein and G. Horton, J. Low Temp. Phys. 9, 151 (1972).

${ }^{11}$ G. Grimvall, B. Magyari-Köpe, V. Ozoliņš, and K. A. Persson, Rev. Mod. Phys. 84, 945 (2012).

${ }^{12}$ P. Souvatzis, O. Eriksson, M. I. Katsnelson, and S. P. Rudin, Phys. Rev. Lett. 100, 095901 (2008).

${ }^{13}$ O. Hellman, I. A. Abrikosov, and S. I. Simak, Phys. Rev. B 84, 180301 (2011).

${ }^{14}$ N. Antolin, O. D. Restrepo, and W. Windl, Phys. Rev. B 86, 054119 (2012).

${ }^{15}$ N. Mounet and N. Marzari, Phys. Rev. B 71, 205214 (2005).

${ }^{16}$ G. D. Mahan, Many-Particle Physics (Plenum Press, New York, 1981).

${ }^{17}$ M. Cardona and M. L. W. Thewalt, Rev. Mod. Phys. 77, 1173 (2005).

${ }^{18}$ P. B. Allen and V. Heine, J. Phys. C 9, 2305 (1976).

${ }^{19}$ P. B. Allen and M. Cardona, Phys. Rev. B 23, 1495 (1981).

${ }^{20}$ F. Giustino, S. G. Louie, and M. L. Cohen, Phys. Rev. Lett. 105, 265501 (2010).

${ }^{21}$ M. Born and R. Oppenheimer, Ann. Phys. 389, 457 (1927).

${ }^{22}$ J. M. Bowman, J. Chem. Phys. 68, 608 (1978).
${ }^{23}$ L. S. Norris, M. A. Ratner, A. E. Roitberg, and R. B. Gerber, J. Chem. Phys. 105, 11261 (1996).

${ }^{24}$ J. O. Jung and R. B. Gerber, J. Chem. Phys. 105, 10332 (1996).

${ }^{25}$ I. Scivetti, N. Gidopoulos, and J. Kohanoff, Phys. Rev. B 78, 224108 (2008).

${ }^{26} \mathrm{H}$. Brooks, Advances in Electronics and Electron Physics, Vol. 7 (Academic Press, New York, 1955).

${ }^{27}$ R. D. King-Smith, R. J. Needs, V. Heine, and M. J. Hodgson, Europhys. Lett. 10, 569 (1989).

${ }^{28}$ P. Allen and J. Hui, Z. Phys. B 37, 33 (1980).

${ }^{29}$ L. D. Landau, L. P. Pitaevskii, E. M. Lifshitz, and A. M. Kosevich, Theory of Elasticity, 3rd ed. (Butterworth-Heinemann, Oxford, 1986).

${ }^{30}$ O. H. Nielsen and R. M. Martin, Phys. Rev. B 32, 3780 (1985).

${ }^{31}$ P. Hohenberg and W. Kohn, Phys. Rev. 136, B864 (1964).

${ }^{32}$ W. Kohn and L. J. Sham, Phys. Rev. 140, A1133 (1965).

${ }^{33}$ D. Vanderbilt, Phys. Rev. B 41, 7892 (1990).

${ }^{34}$ S. J. Clark, M. D. Segall, C. J. Pickard, P. J. Hasnip, M. I. J. Probert, K. Refson, and M. C. Payne, Z. Kristallogr. 220, 567 (2005).

${ }^{35}$ H. J. Monkhorst and J. D. Pack, Phys. Rev. B 13, 5188 (1976).

${ }^{36}$ D. M. Ceperley and B. J. Alder, Phys. Rev. Lett. 45, 566 (1980).

${ }^{37}$ J. P. Perdew and A. Zunger, Phys. Rev. B 23, 5048 (1981).

${ }^{38}$ J. P. Perdew, K. Burke, and M. Ernzerhof, Phys. Rev. Lett. 77, 3865 (1996).

${ }^{39}$ H. Wendel and R. M. Martin, Phys. Rev. Lett. 40, 950 (1978).

${ }^{40}$ P. Giannozzi, S. de Gironcoli, P. Pavone, and S. Baroni, Phys. Rev. B 43, 7231 (1991).

${ }^{41}$ S. Baroni, S. de Gironcoli, A. Dal Corso, and P. Giannozzi, Rev. Mod. Phys. 73, 515 (2001).

${ }^{42}$ C. Cohen-Tannoudji, B. Diu, and F. Laloë, Quantum Mechanics, Vol. 2 (John Wiley \& Sons, Paris, 1977).

${ }^{43}$ S. J. Nolan, M. J. Gillan, D. Alfè, N. L. Allan, and F. R. Manby, Phys. Rev. B 80, 165109 (2009).

${ }^{44}$ C. D. Clark, P. J. Dean, and P. V. Harris, Proc. R. Soc. London A 277, 312 (1964).

${ }^{45}$ M. Cardona, Solid State Commun. 133, 3 (2005). 
${ }^{46}$ S. Zollner, M. Cardona, and S. Gopalan, Phys. Rev. B 45, 3376 (1992).

${ }^{47}$ M. L. Cohen and T. K. Bergstresser, Phys. Rev. 141, 789 (1966).

${ }^{48}$ L. Artus and Y. Bertrand, Solid State Commun. 61, 733 (1987).

${ }^{49}$ J. Bhosale, A. K. Ramdas, A. Burger, A. Muñoz, A. H. Romero, M. Cardona, R. Lauck, and R. K. Kremer, Phys. Rev. B 86, 195208 (2012).

${ }^{50}$ R. W. Godby, M. Schlüter, and L. J. Sham, Phys. Rev. Lett. 56, 2415 (1986).
${ }^{51}$ R. W. Godby, M. Schlüter, and L. J. Sham, Phys. Rev. B 37, 10159 (1988).

${ }^{52}$ F. Gygi and A. Baldereschi, Phys. Rev. Lett. 62, 2160 (1989).

${ }^{53}$ E. Cannuccia and A. Marini, Phys. Rev. Lett. 107, 255501 (2011).

${ }^{54}$ E. Cannuccia and A. Marini, Eur. Phys. J. B 85, 320 (2012).

${ }^{55}$ G. A. Slack and S. F. Bartram, J. Appl. Phys. 46, 89 (1975).

${ }^{56}$ B. W. James and H. Kheyrandish, J. Phys. C 15, 6321 (1982).

${ }^{57}$ W. M. C. Foulkes, L. Mitas, R. J. Needs, and G. Rajagopal, Rev. Mod. Phys. 73, 33 (2001). 